\title{
LPPR2 expression associates with survival in triple negative breast cancer.
}

Shahan Mamoor, $\mathrm{MS}^{1}$

${ }^{1}$ shahanmamoor@gmail.com

East Islip, NY USA

We mined published microarray data (1) to understand the most significant gene expression differences in the tumors of triple negative breast cancer patients based on survival following treatment: dead or alive. We observed significant transcriptome-wide differential expression of lipid phosphate phosphatase-related protein type 2, encoded by LPPR2 when comparing the primary tumors of triple negative breast cancer patients dead or alive. LPPR2 may be of relevance as a biomarker or as a molecule of interest in understanding the etiology or progression of triple negative breast cancer.

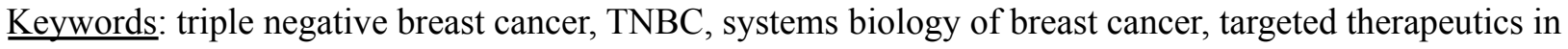
breast cancer, LPPR2, lipid phosphate phosphatase-related protein type 2. 
Triple negative breast cancer is defined by lack of expression of the receptors for the hormones progesterone and estrogen (PR negative and ER negative), as well as for the human epidermal growth factor receptor 2 (HER2 negative) $(2,3)$. Though not completely identical, triple negative breast cancer shares significant overlap with the basal or basal-like molecular subtype of human breast cancer (3). TNBC is more frequently diagnosed in women of African descent (black women) for reasons not understood, and their disease course is similarly more aggressive than in white women with triple negative breast cancer $(2,3)$. To understand the transcriptional biology of triple negative breast cancer in an unbiased fashion and at the systems level, we mined published microarray data paired with patient survival data (1), performing comparative transcriptome analysis based on survival at time of data collection: dead or alive. We present here a finding from the results of this analysis.

\section{Methods}

We used dataset GSE142102 (1) for this differential gene expression analysis in conjunction with GEO2R. GSE142102 was generated using Affymetrix Human Gene 2.1 ST Array technology; in this analysis, we used data from $n=87$ tumors from TNBC patients that were dead at time of analysis and $n=123$ tumors from TNBC patients that were alive at time of analysis. The Benjamini and Hochberg method of $\mathrm{p}$-value adjustment was used for ranking of differential expression but raw $p$-values were used to assess statistical significance of global differential expression. Log-transformation of data was auto-detected, and the NCBI generated category of platform annotation was used. A statistical test was performed to evaluate whether LPPR2 expression was significantly different between the primary tumors of TNBC patients that were dead or alive using a two-tailed t-test.

\section{Results}

We performed comparative transcriptome analysis using published microarray data (1) to understand in an unbiased fashion the most significant gene expression differences in the primary tumors of patients with triple negative breast cancer based on survival outcomes.

$\underline{\text { LPR2 } 2 \text { is differentially expressed in triple negative breast cancer and negatively correlates with survival. }}$

When comparing primary tumors of 87 live patients with triple negative breast cancer with that of 123 dead TNBC patients we identified lipid phosphate phosphatase-related protein type 2, LPPR2, as among the most significant transcriptional differences based on survival in human triple negative breast cancer. When ranking each of the transcripts whose expression was measured by microarray based on significance of difference in expression between the tumors of patients dead and alive, LPPR2 ranked 103 out of 53617 total transcripts, equivalent to $99.8 \%$ differential expression (Chart 1). LPPR2 differential expression in the tumors of patients based on survival outcomes was statistically significant (Figure 1; $p=0.00253741)$.

LPPR2 is expressed at significantly lower levels in the breast tumors of TNBC patients when comparing based on survival.

We obtained exact mRNA expression levels for LPPR2 from breast tumors of TNBC patients dead and alive to understand the magnitude and direction of LPPR2 expression change based on survival outcomes. LPPR2 was expressed at lower levels in tumors of TNBC patients alive as compared to that of TNBC patients dead (Figure 1). Decreased expression of LPPR2 in primary breast tumors was statistically 


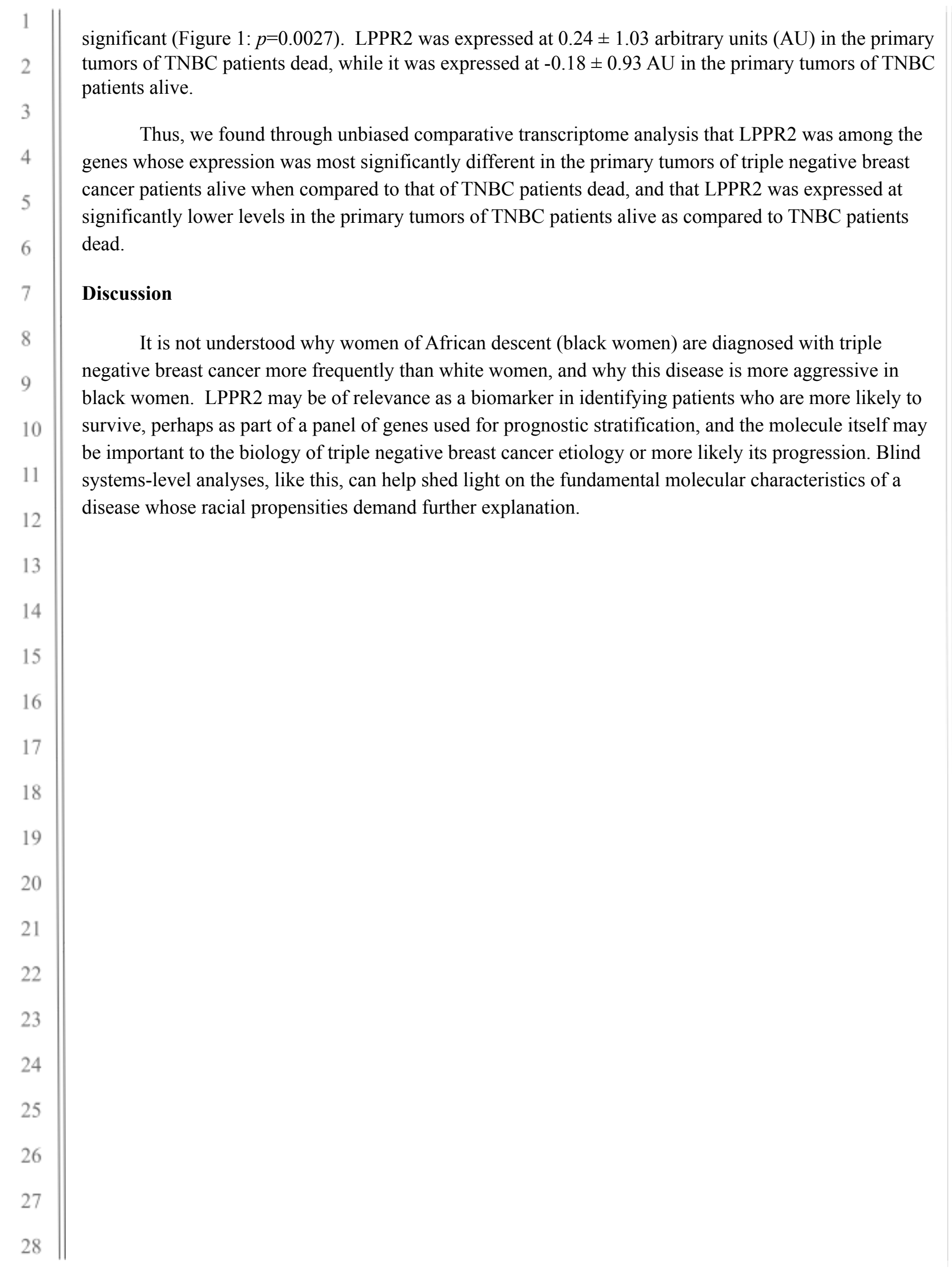




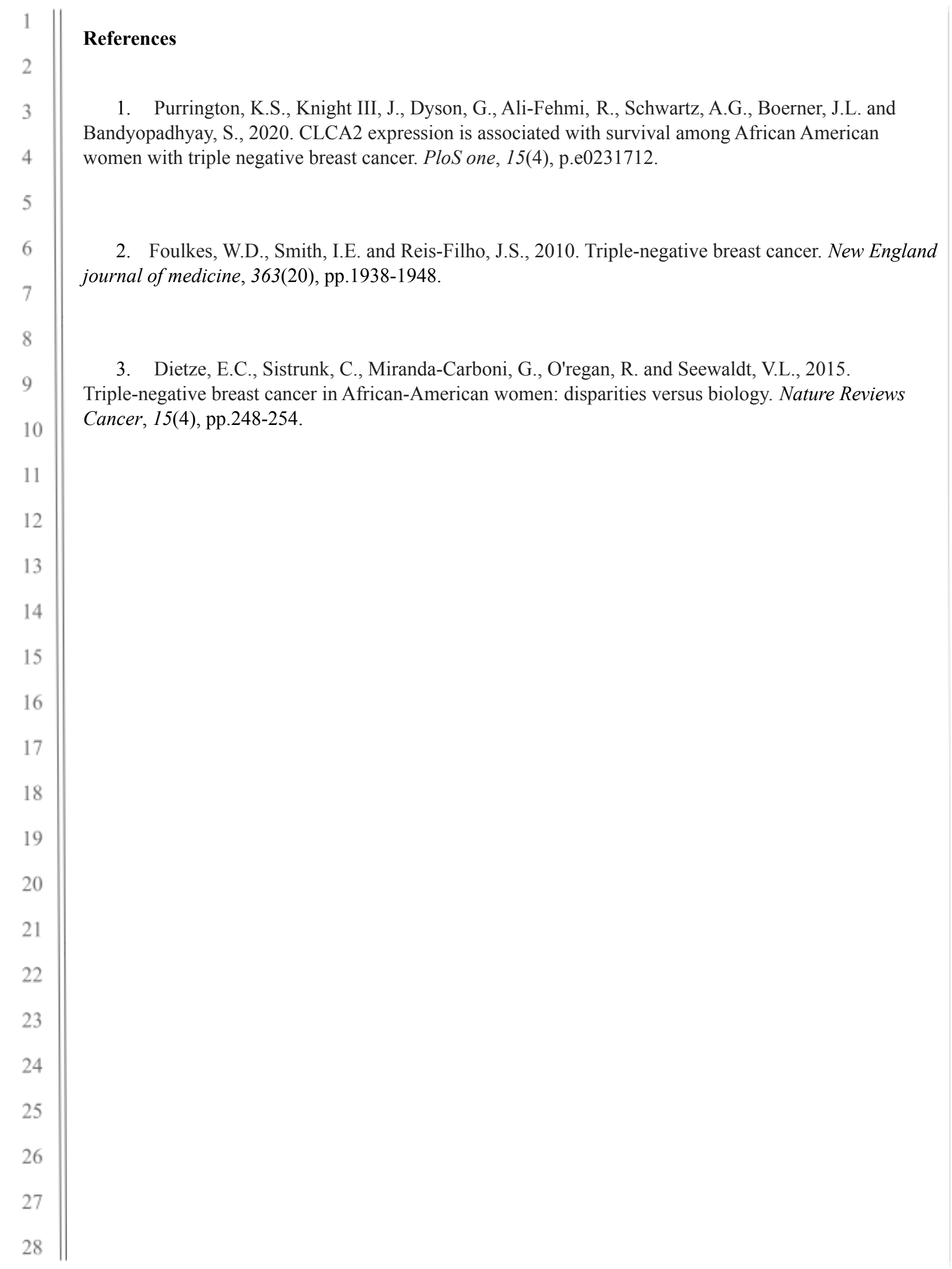




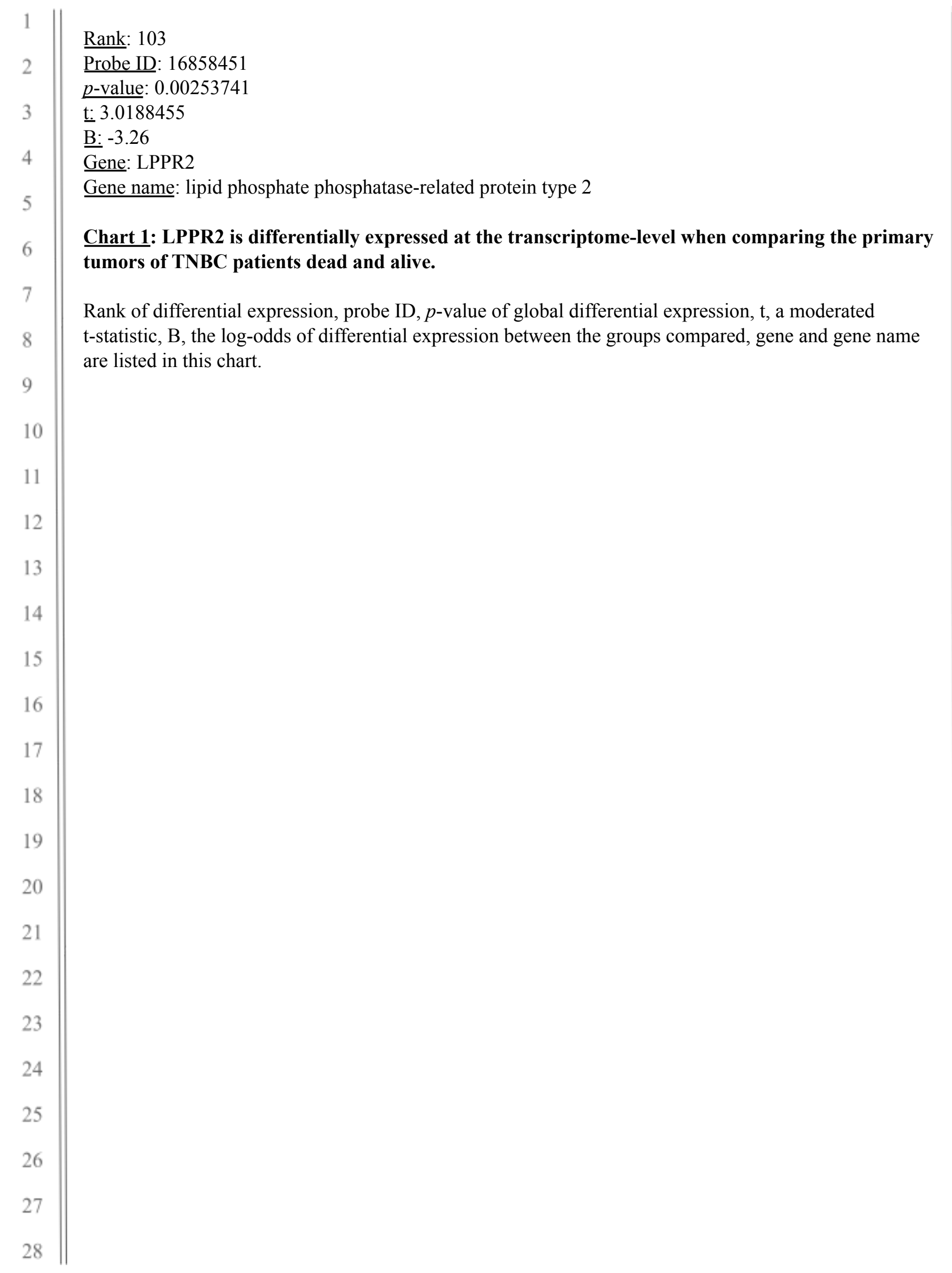




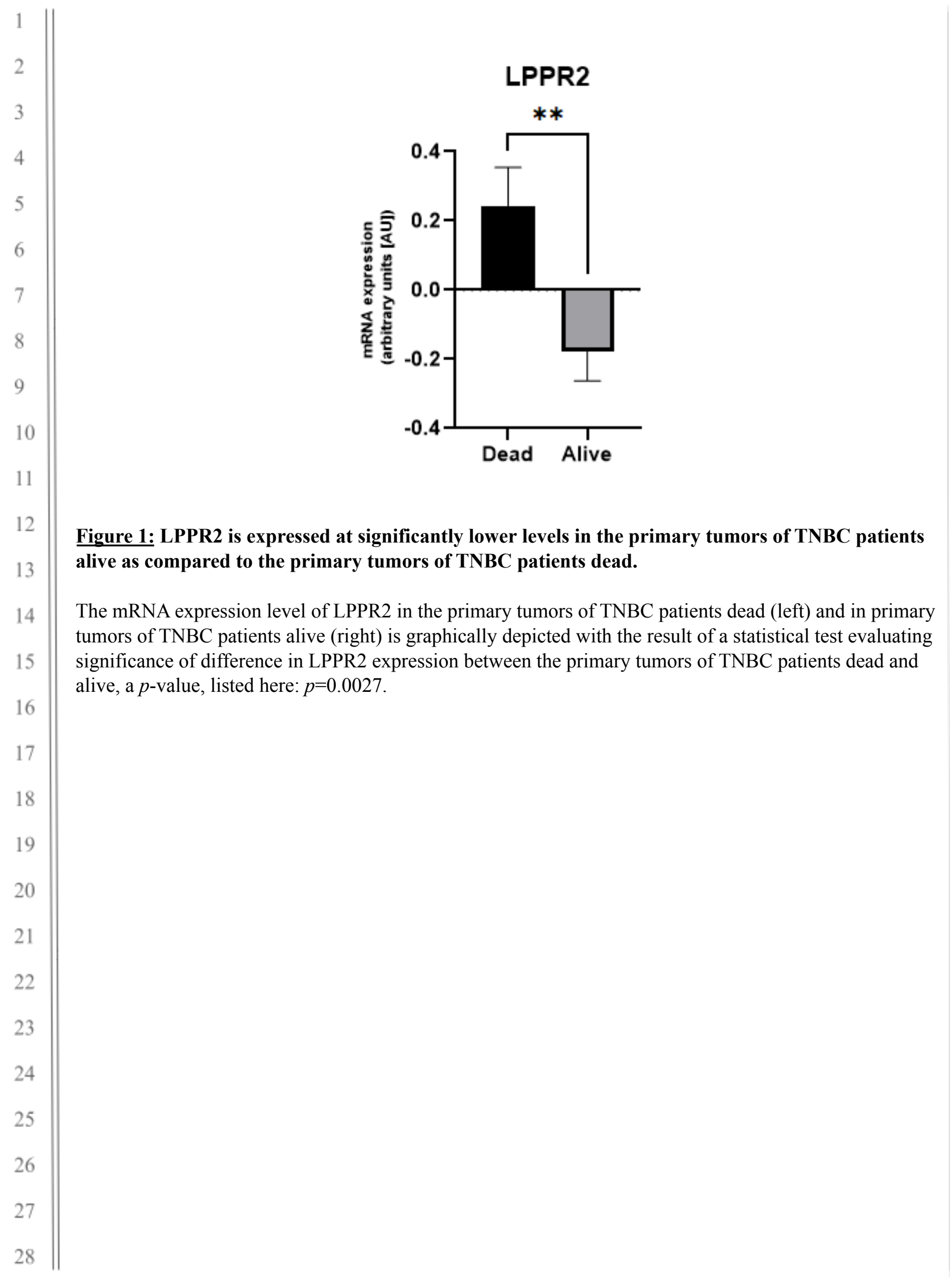

